Supplementary information for

\title{
Stretchable, Self-healing and Skin-Mounted Active Sensor for Multipoint Muscle Function Assessment
}

Chan Wang, ${ }^{\dagger, t, *}$ Xuecheng Qu, ${ }^{+,+, *}$ Qiang Zheng, ${ }^{\dagger,+, *}$ Ying Liu, ${ }^{\dagger,+}$ Puchuan Tan, ${ }^{\dagger, \xi}$ Bojing Shi, ${ }^{\dagger, \xi}$ Han Ouyang, ${ }^{\dagger, \xi}$ Shengyu Chao, ${ }^{\dagger,+}$ Yang Zou, ${ }^{\dagger, t}$ Chaochao Zhao, ${ }^{\dagger, \perp}$ Zhuo Liu, ${ }^{\dagger, \S}$

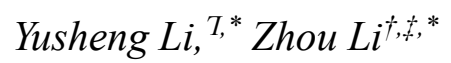

${ }^{\dagger}$ CAS Center for Excellence in Nanoscience, Beijing Key Laboratory of Micro-nano Energy and Sensor, Beijing Institute of Nanoenergy and Nanosystems, Chinese Academy of Sciences, Beijing, 101400, China

${ }^{*}$ School of Nanoscience and Technology, University of Chinese Academy of Sciences, Beijing, 100049, China

${ }^{\S}$ Beijing Advanced Innovation Centre for Biomedical Engineering, Key Laboratory for Biomechanics and Mechanobiology of Ministry of Education, School of Biological Science and Medical Engineering, Beihang University, Beijing, 100191, China

${ }^{\perp}$ Department of Biomedical Engineering, School of Medical Engineering, Foshan University, Foshan, 528225, China

${ }^{\mathrm{T}}$ Department of Orthopedics, Xiangya Hospital, Central South University, Changsha 410008, China

${ }^{*}$ Corresponding Authors:

E-mails: liyusheng@csu.edu.cn; zli@binn.cas.cn 


\section{The Supplementary Materials include:}

Supplementary Figure 1-17.

Supplementary Note 1-3.

Supplementary Movie 1-7.

Movie S1: The self-healing ability of the electrode.

Movie S2: The detect limit of TSAS.

Movie S3: The self-healing ability of TSAS.

Movie S4: The relationship of $\mathrm{V}_{\text {oc }}$ and force.

Movie S5: TSAS used in muscle function assessment (male).

Movie S6: TSAS used in muscle function assessment (female).

Movie S7: TSAS used in real-time muscle function assessment. 


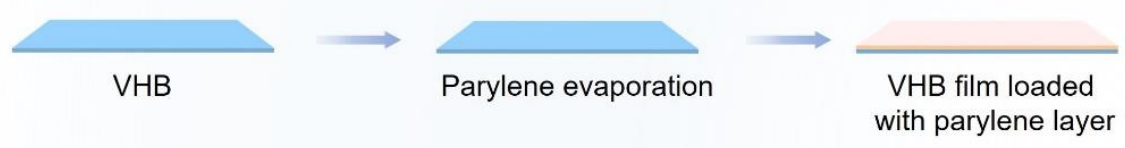

b
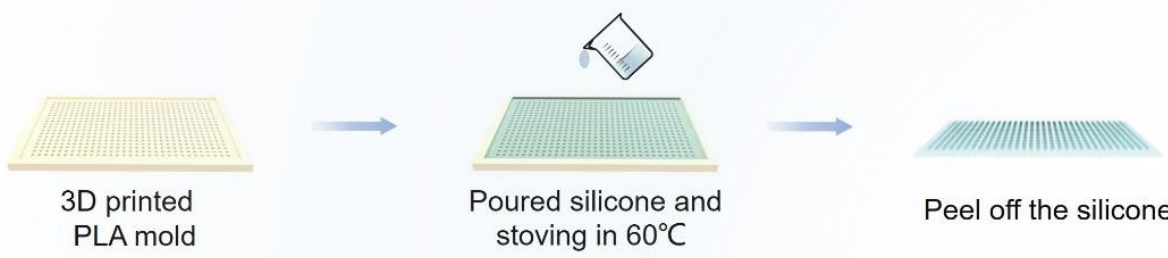

stoving in $60^{\circ} \mathrm{C}$

Peel off the silicone

Figure S1. Schematic diagram about the preparation process of friction layer. Parylene layer loading on the VHB (a) and silicone layer (b).

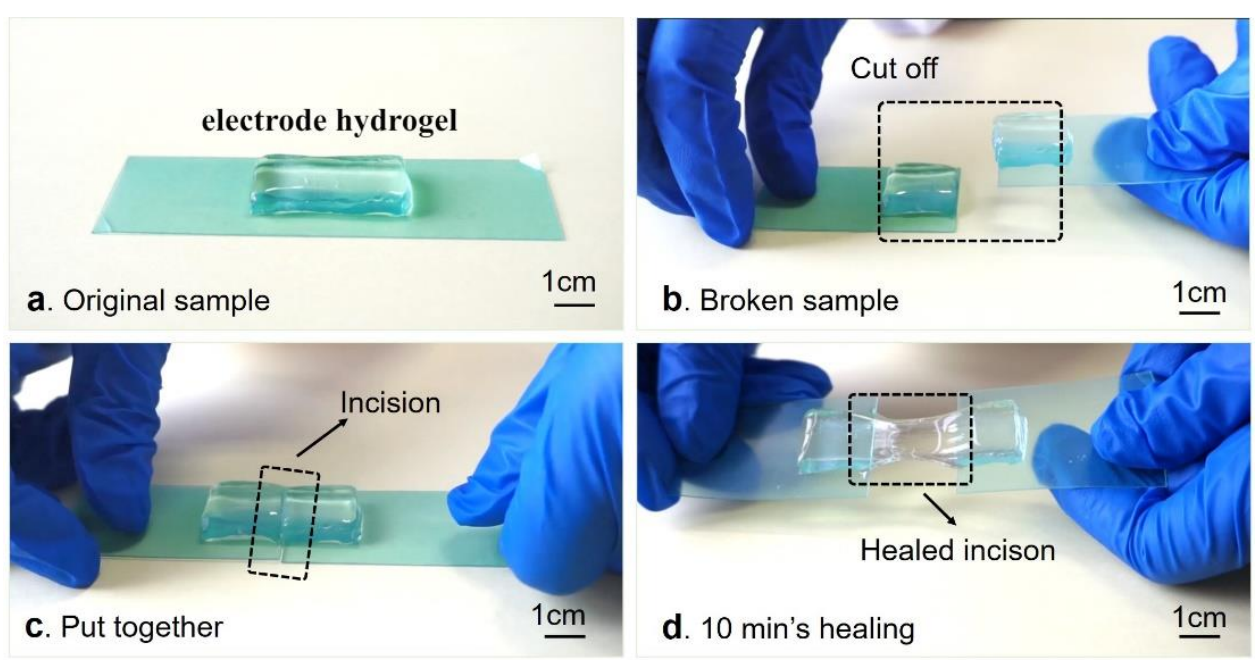

Figure S2. The healing process of hydrogel electrode. (a) The original simple. (b) The hydrogel was cut in half with a scissor. (c) Bring two parts together slightly. (d) After 10 min's healing, the healed notch could afford big deformation without rupture. Scale bar: $1 \mathrm{~cm}$. 

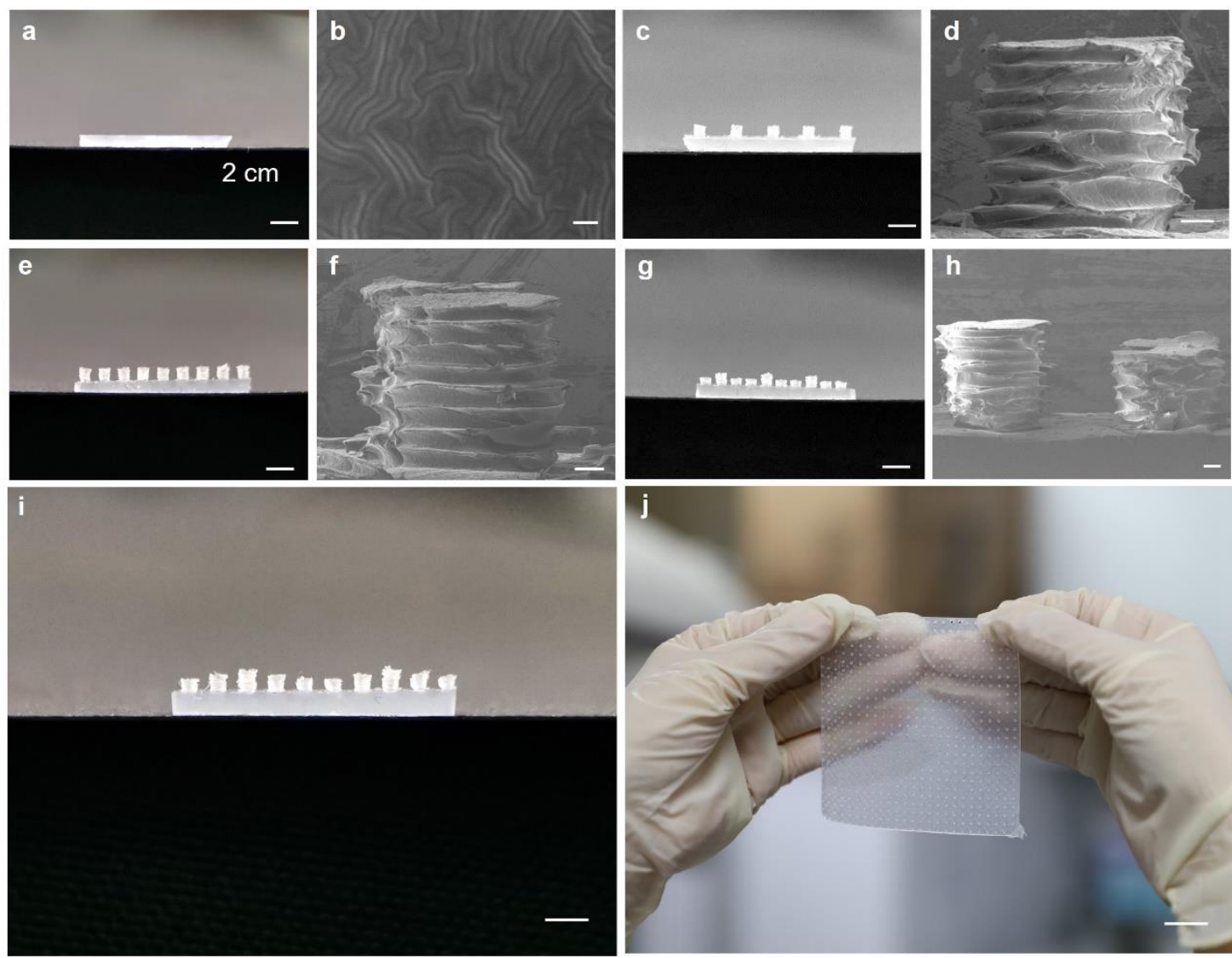

Figure S3. The SEM and optical images of silicone film are used as frictional layer. (a, b) The silicon film with no surface microstructure. Scale bar: $2 \mathrm{~mm}, 10 \mu \mathrm{m}$. (c, d) The silicon film with loose microcolumn (spacer). Microcolumns' hight: $0.8 \mathrm{~mm}$. Distance between two microcolumns: $3 \mathrm{~mm}$. Scale bar: $3 \mathrm{~mm}, 200 \mu \mathrm{m}$. (e, f) The silicon film with compact microcolumn (spacer). Microcolumns' hight: $0.8 \mathrm{~mm}$. Distance between two microcolumns: $1 \mathrm{~mm}$. Scale bar: $2 \mathrm{~mm}, 200 \mu \mathrm{m}$. (g, h) The silicon film with interphase microcolumn (spacer). Microcolumns' hight: 0.8 and $0.4 \mathrm{~mm}$. Distance between two microcolumns: $0.4 \mathrm{~mm}$. Scale bar: $2 \mathrm{~mm}, 200 \mu \mathrm{m}$. (i) The silicon film with surface microcolumns arranged in a wavy pattern. Scale bar: $2 \mathrm{~mm}$. (j) The optical image of the silicon film. Scale bar: $2 \mathrm{~cm}$. 


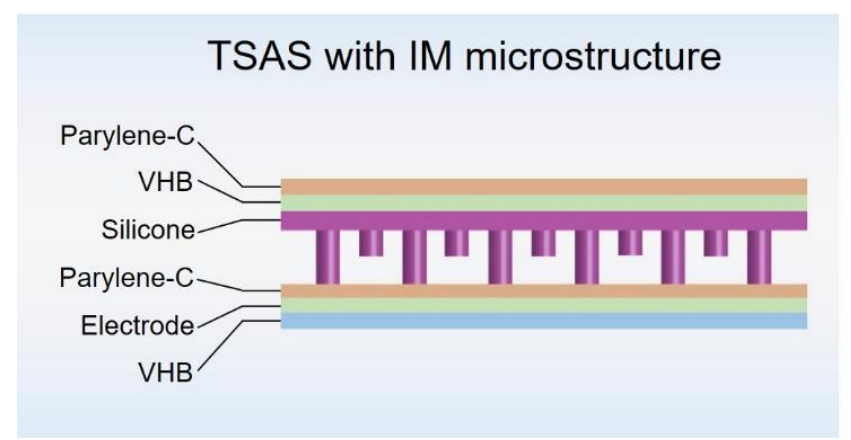

Figure S4. The assembly layers of the TSAS with IM microstructure.
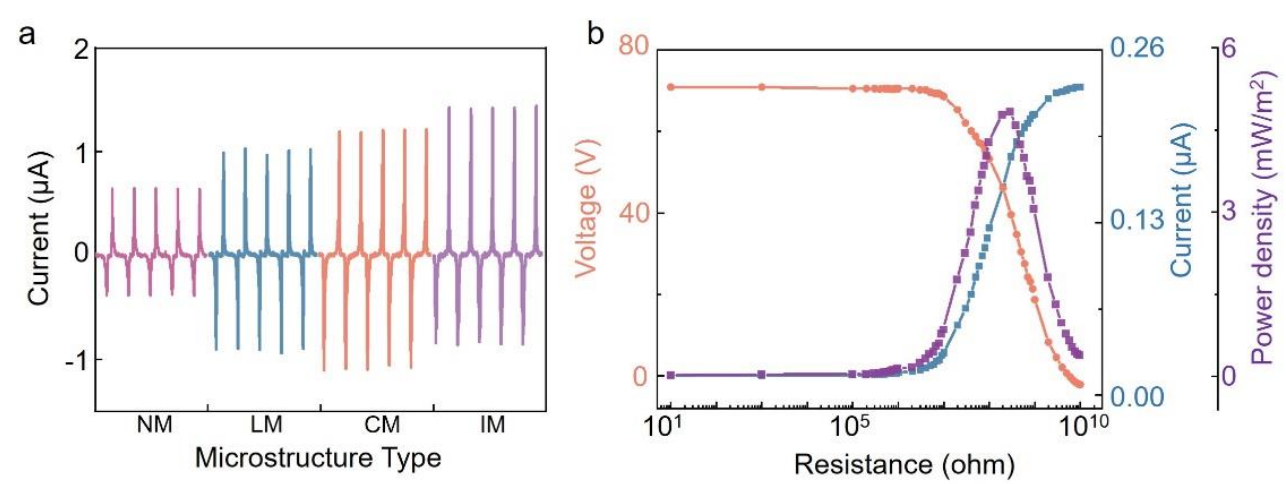

Figure S5. Electrical characteristics of TSAS. (a) $\mathrm{I}_{\mathrm{sc}}$ of the TSAS with four types of frictional layer. (b) $\mathrm{V}_{\mathrm{oc}} / \mathrm{I}_{\mathrm{sc}}$ and peak power density of the TSAS-IM along with the loading resistance.
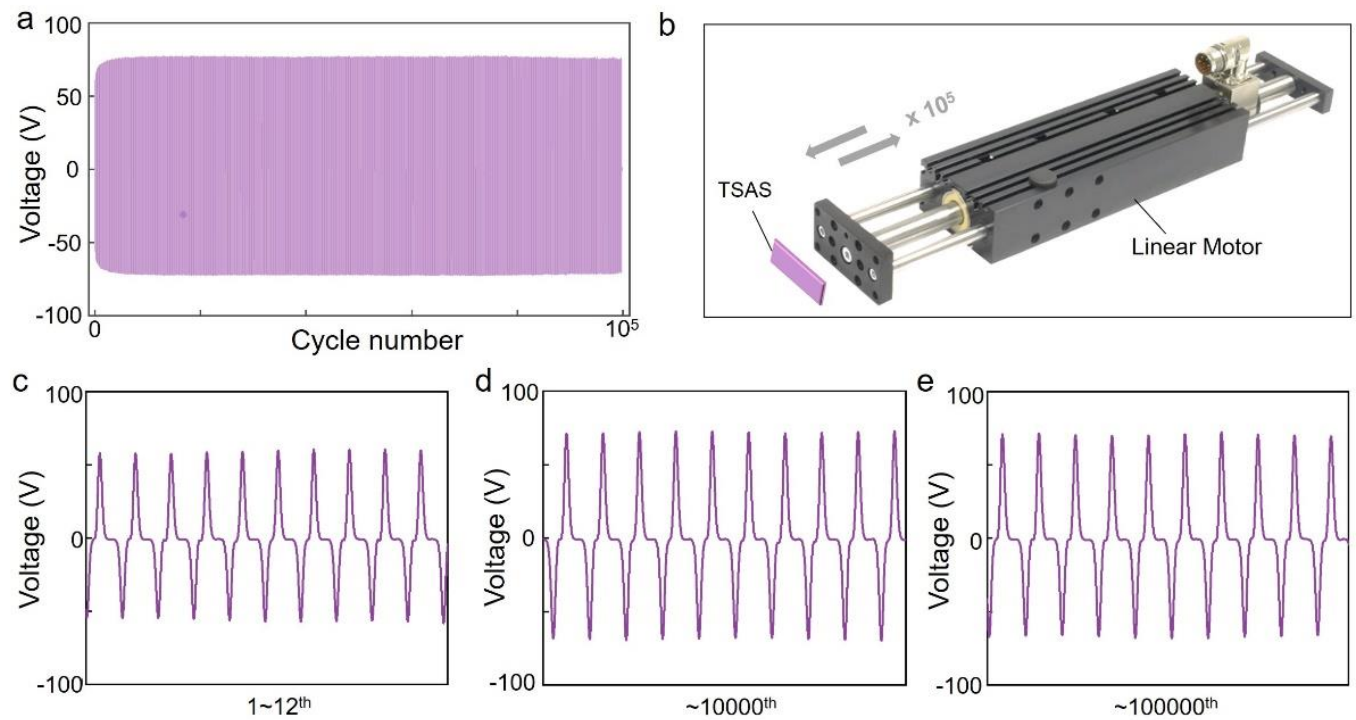

Figure S6. The tensile fatigue resistance test of the TSAS. (a) $V_{\text {oc }}$ of TSAS that lasted for $\sim 10^{5}$ cycles. (b) Schematic diagram of the equipment used to test open-circuit voltage. The details of voltage curves at the $1 \sim 12^{\text {th }}$ cycle, around $10000^{\text {th }}$ cycle and at the end of test are shown in (c-e), respectively. 


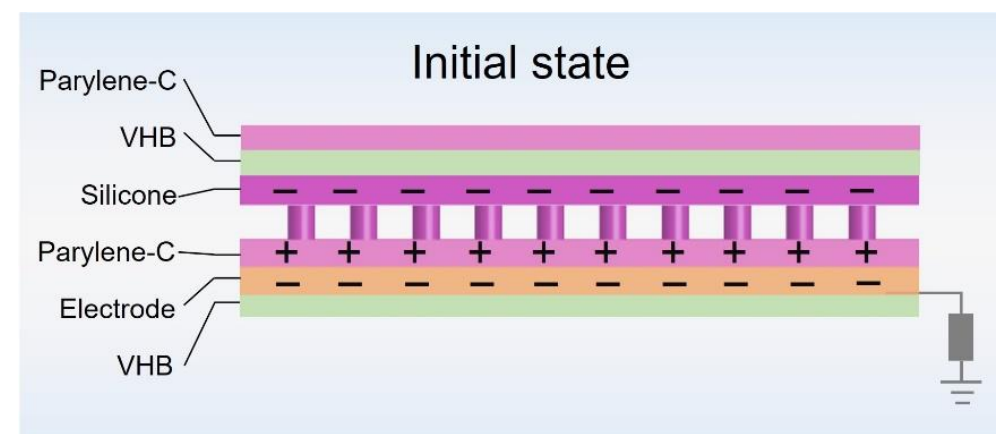

Figure S7. The full assembly layers of the TSAS.

a

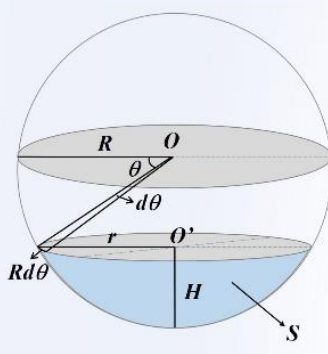

b

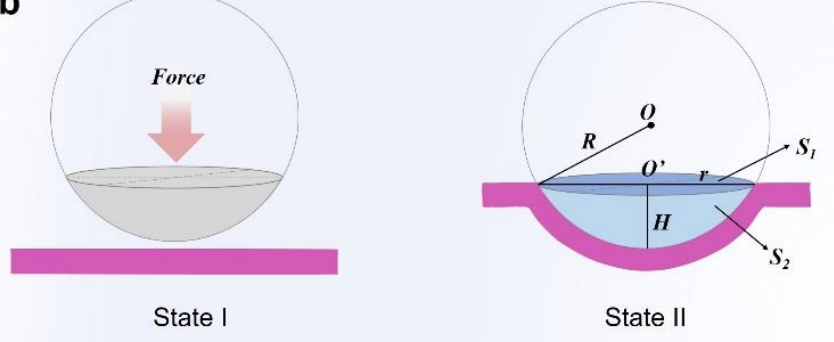

Figure S8. Schematic diagram of spherical crown's surface area (a) and the contacted area change of TSAS's two frictional layers (b).

Note S1. Equation derivation of the surface area $(\mathrm{S})$ of the spherical crown and the contacted area change of TSAS's two frictional layers.

$$
\begin{aligned}
S & =\int_{\theta}^{\frac{\pi}{2}} 2 \pi r R \cdot d \theta \\
& =\int_{\theta}^{\frac{\pi}{2}} 2 \pi R^{2} \cos \theta \cdot d \theta \\
& =2 \pi R^{2} \int_{\theta}^{\frac{\pi}{2}} \cos \theta \cdot d \theta \\
& =2 \pi R^{2}(1-\sin \theta) \\
& =2 \pi R H
\end{aligned}
$$

The surface area (S) of the spherical crown is deduced as bellow:

The parameters, like: $\theta, r, R, H$ could be found in Supplementary Fig. 5a.

The contacted area change $(\Delta \mathrm{S})$ of TSAS during force applying calculated as: 


$$
\begin{aligned}
\Delta S & =S_{2}-S_{1} \\
& =2 \pi R H-\pi r^{2} \\
& =2 \pi R H-\pi\left[R^{2}-(R-H)^{2}\right] \\
& =2 \pi R H-2 \pi R H+\pi H^{2} \\
& =\pi H^{2}
\end{aligned}
$$

Note S2. The calculation process of equation about $\mathrm{V}_{\text {oc }}$ and $\Delta \mathrm{S}$ through method of undetermined coefficients.

Choose two groups dates from Table $\mathrm{S} 1 . \Delta \mathrm{S}=314, \mathrm{~V}_{\mathrm{oc}}=0.79362 ; \Delta \mathrm{S}=706.5, \mathrm{~V}_{\mathrm{oc}}=$ 1.713;

$$
\begin{aligned}
& 0.79362=314 \times \mathrm{k}_{1}+\mathrm{k}_{2} \\
& 1.713=706.5 \times \mathrm{k}_{1}+\mathrm{k}_{2}
\end{aligned}
$$

The $k_{1}, k_{2}$ were calculated to be 0.00234 and 0.005886 , respectively.

Thus,

$$
\mathrm{V}_{\mathrm{oc}}=0.00234 \times \Delta \mathrm{S}+0.005886
$$

The equation curve shows good coincidence with experimental data in Table S1. Shown as below:

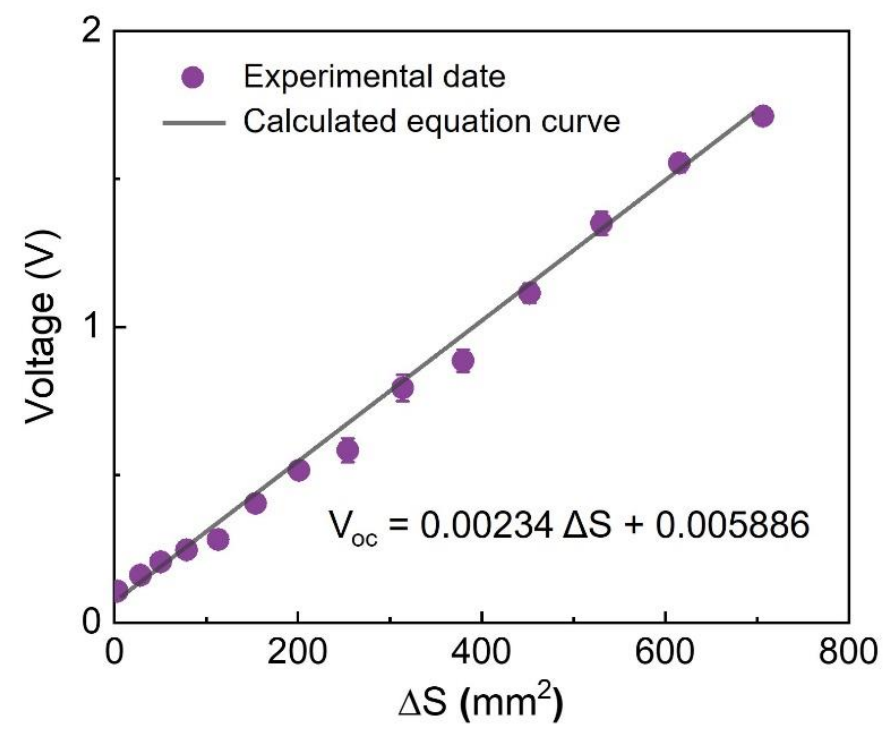

Figure S9. The relationship of $\mathrm{V}_{\mathrm{oc}}$ and $\Delta \mathrm{S}$. The experimental data and equation curve were represented by scatters and solid line, respectively. 


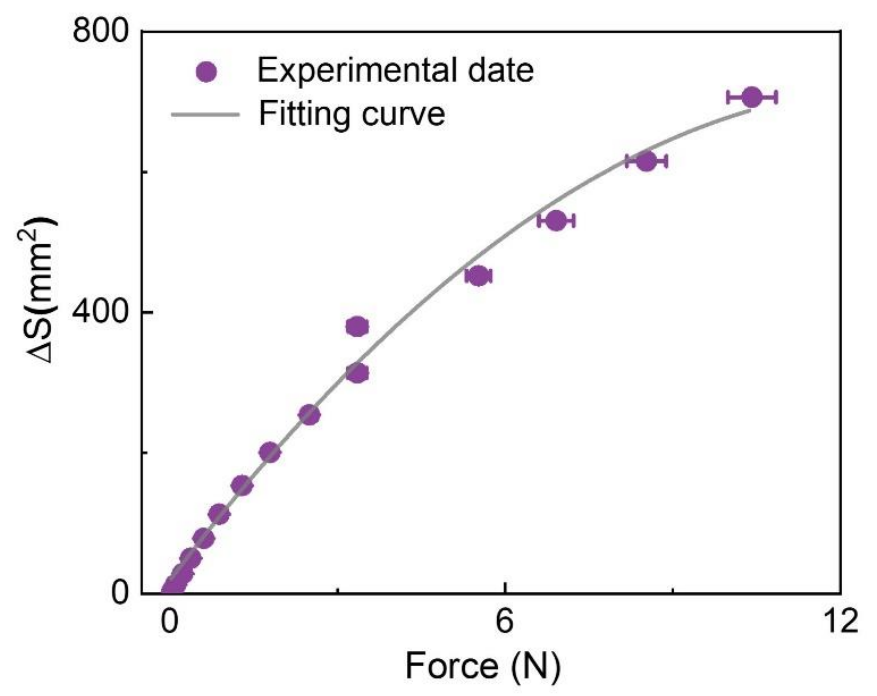

Figure S10. The relationship of $\Delta \mathrm{S}$ and $\mathrm{F}$. The experimental data and fitting curve were represented by scatters and solid line, respectively.

Note S3. The calculation process of the equation about $V_{o c}$ and $F$.

Above, the equation of

$$
\begin{aligned}
& \mathrm{V}_{\mathrm{oc}}=0.00234 \times \Delta \mathrm{S}+0.005886 \\
& \Delta \mathrm{S}=14.14+105.4 \mathrm{~F}-3.96 \mathrm{~F}^{2}
\end{aligned}
$$

were obtained. Then, the equation about $\mathrm{V}$ and $\mathrm{F}$ can be calculated through the substitution method as:

$$
\mathrm{V}_{\mathrm{oc}}=0.085+0.247 \mathrm{~F}-0.00919 \mathrm{~F}^{2}
$$

The equation curve shows good coincidence with experimental data in Table 1. Shown as below: 


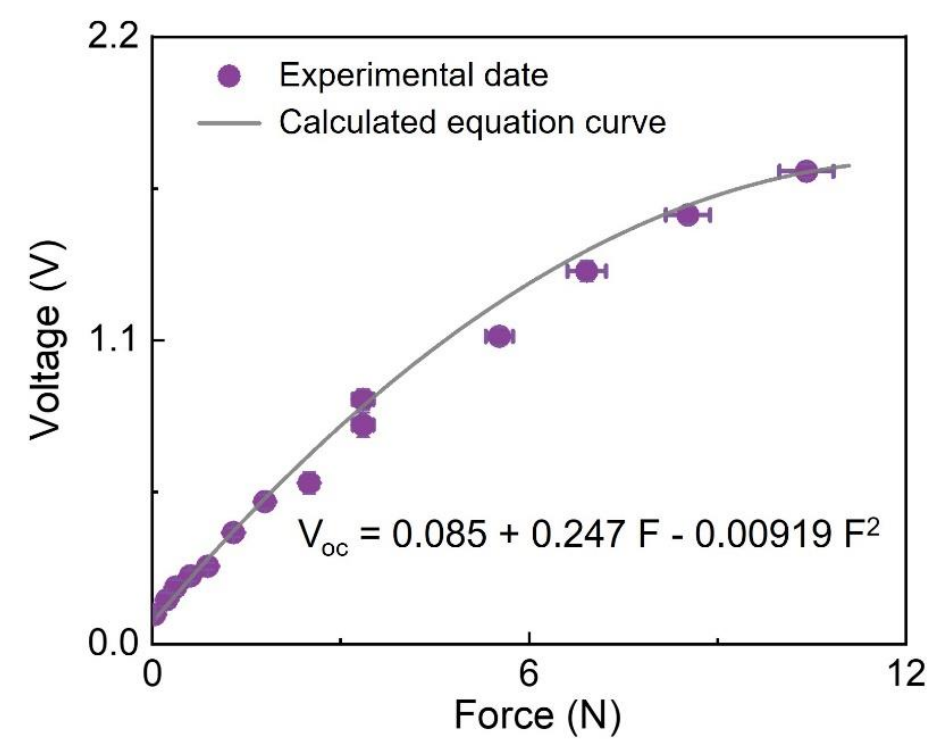

Figure S11. The relationship of $\mathrm{V}_{\mathrm{oc}}$ and $\mathrm{F}$. The experimental data and calculated equation curve were represented by scatters and solid line, respectively.

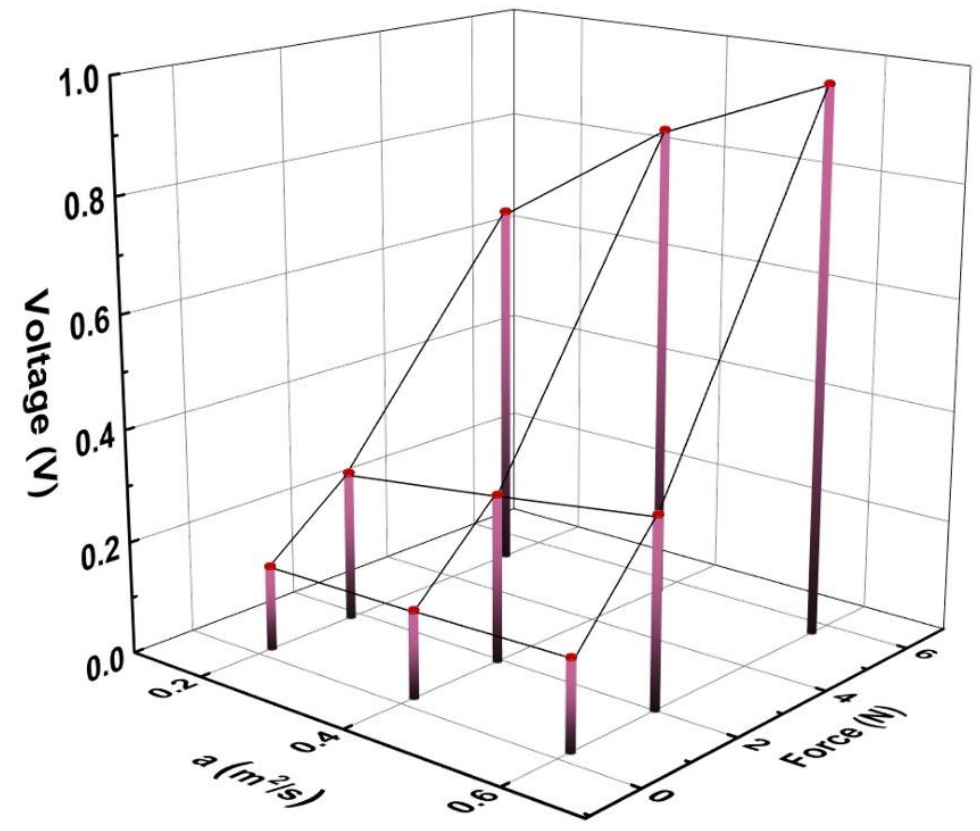

Figure S12. Effects of acceleration and force on open-circuit voltage of TSAS. 

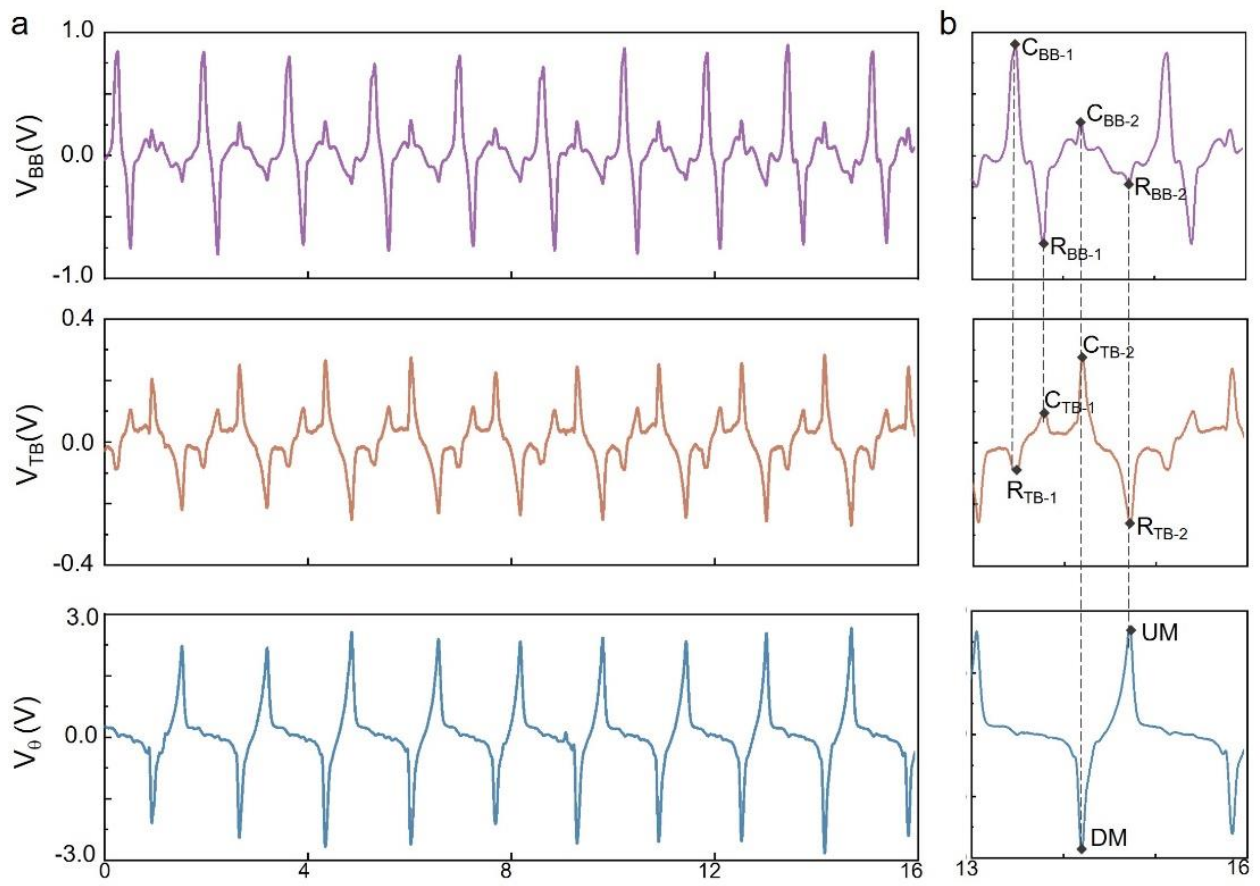

Figure S13. TSAS used for muscle function monitoring for male volunteer 1. (a) The $\mathrm{V}_{\mathrm{oc}}$ of TSAS adhered on biceps brachii $\left(\mathrm{V}_{\mathrm{BB}}\right)$, triceps brachii $\left(\mathrm{V}_{\mathrm{TB}}\right)$ and elbow $\left(\mathrm{V}_{\theta}\right)$, respectively. Details about voltage curve of $\mathrm{V}_{\mathrm{BB}}, \mathrm{V}_{\mathrm{TB}}, \mathrm{V}_{\theta}$ were shown in (b).
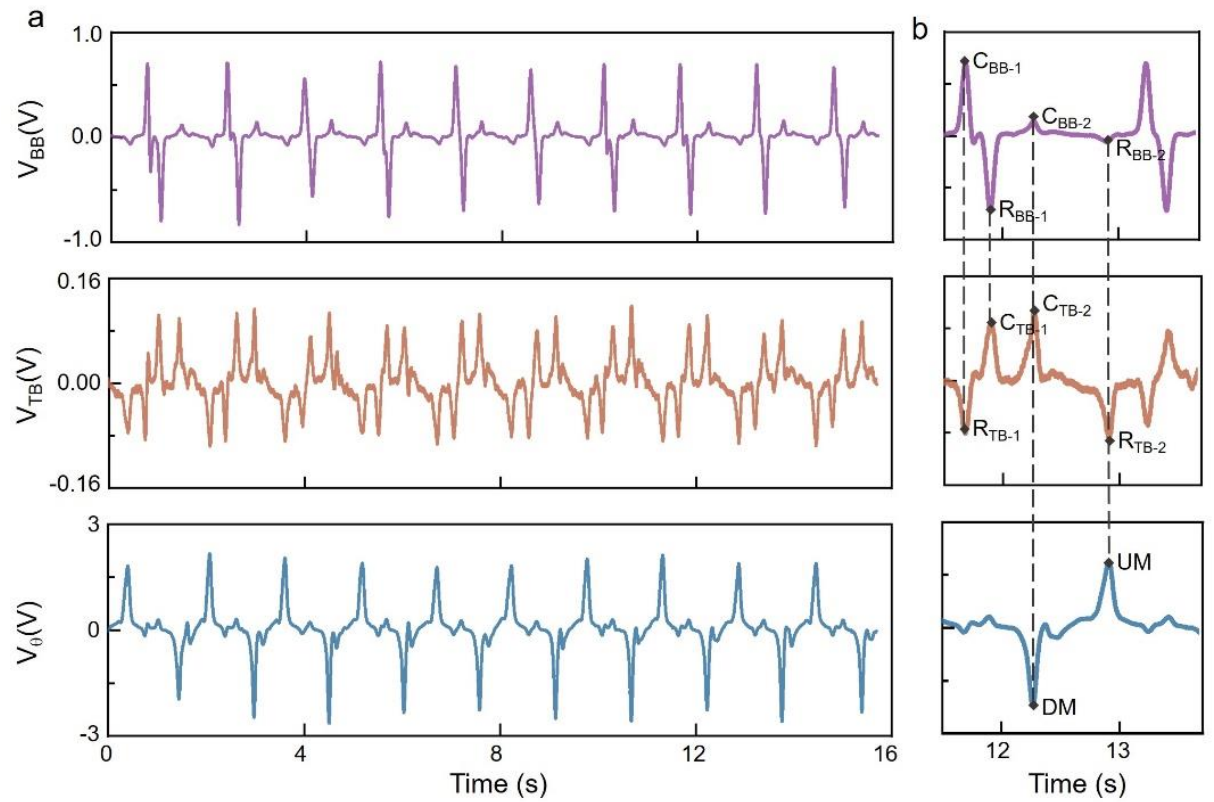

Figure S14. TSAS used for muscle function monitoring for female volunteer 2. (a) The $\mathrm{V}_{\text {oc }}$ of TSAS adhered on biceps brachii $\left(\mathrm{V}_{\mathrm{BB}}\right)$, triceps brachii $\left(\mathrm{V}_{\mathrm{TB}}\right)$ and elbow $\left(\mathrm{V}_{\theta}\right)$, respectively. Details about voltage curve of $\mathrm{V}_{\mathrm{BB}}, \mathrm{V}_{\mathrm{TB}}, \mathrm{V}_{\theta}$ were shown in (b). 


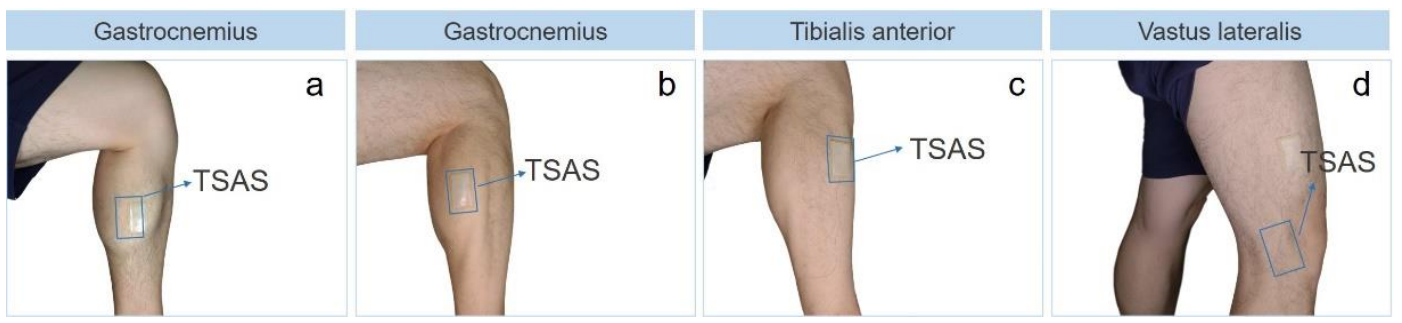

Figure S15. The TSAS used for monitoring other muscle groups. Gastrocnemius $(a, b)$, tibialis anterior (c) and vastus lateralis (d).
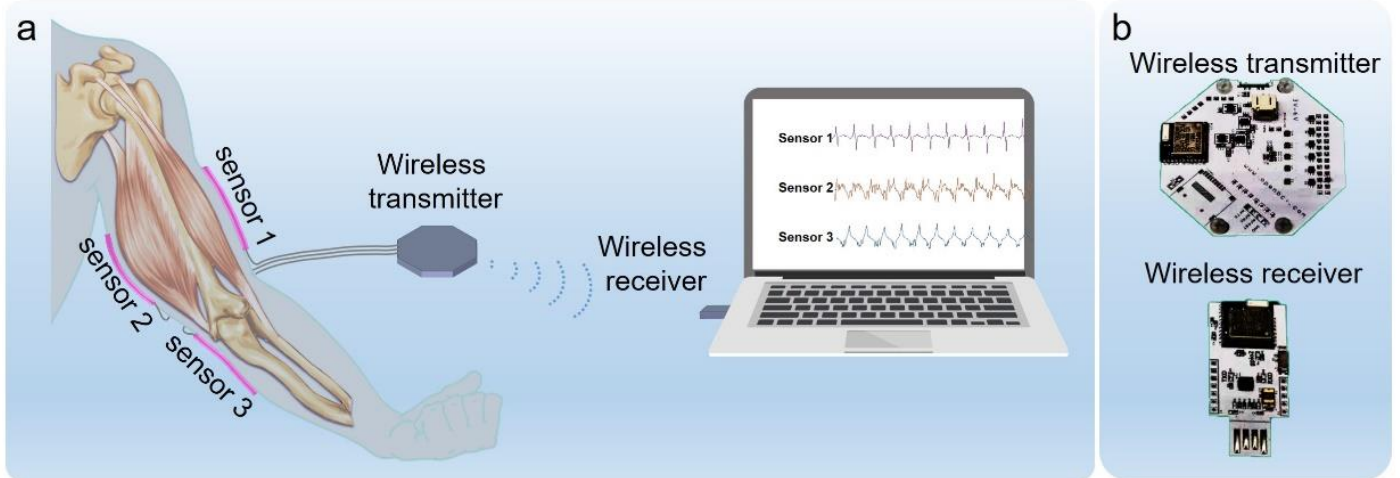

Figure S16. The TSAS is used in real-time muscle function assessment. (a) Schematic diagram of wireless transmission system used in online muscle function assessment. (b) The photos of wireless transmitter and receiver.
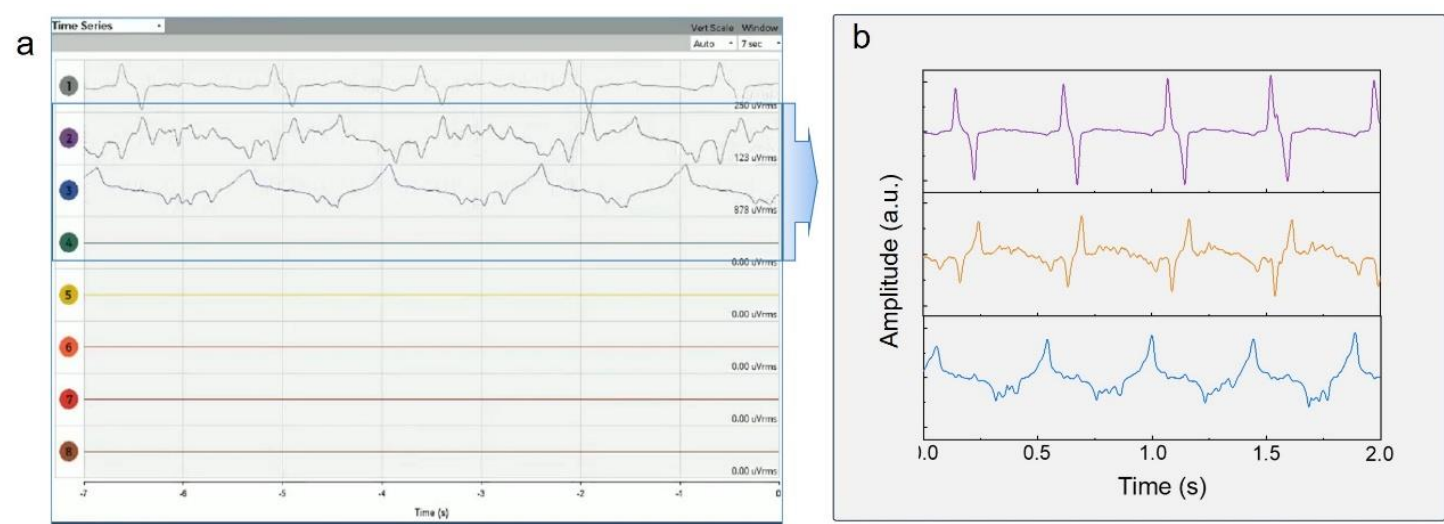

Figure S17. (a) The visual terminal interfaces. The real-time dates of channels 1, 2 and 3 are from TSAS equipped on triceps brachii, biceps brachii and elbow. (b) The realtime dates are recorded by channel 1,2 and 3 . 